\title{
Observation of spontaneous Brillouin cooling
}

\author{
Gaurav Bahl ${ }^{1 \star}$, Matthew Tomes ${ }^{1}$, Florian Marquardt ${ }^{2,3}$ and Tal Carmon ${ }^{1}$
}

\begin{abstract}
Although bolometric- and ponderomotive-induced deflection of device boundaries are widely used for laser cooling, the electrostrictive Brillouin scattering of light from sound was considered an acousto-optical amplification-only process ${ }^{1-7}$. It was suggested that cooling could be possible in multiresonance Brillouin systems $\mathrm{s}^{5-8}$ when phonons experience lower damping than light ${ }^{8}$. However, this regime was not accessible in electrostrictive Brillouin systems ${ }^{1-3,5,6}$ as backscattering enforces high acoustical frequencies associated with high mechanical damping ${ }^{1}$. Recently, forward Brillouin scattering ${ }^{3}$ in microcavities ${ }^{7}$ has allowed access to low-frequency acoustical modes where mechanical dissipation is lower than optical dissipation, in accordance with the requirements for cooling ${ }^{8}$. Here we experimentally demonstrate cooling via such a forward Brillouin process in a microresonator. We show two regimes of operation for the electrostrictive Brillouin process: acoustical amplification as is traditional and an electrostrictive Brillouin cooling regime. Cooling is mediated by resonant light in one pumped optical mode, and spontaneously scattered resonant light in one anti-Stokes optical mode, that beat and electrostrictively attenuate the Brownian motion of the mechanical mode.
\end{abstract}

Spontaneous Raman and Brillouin scattering are common to almost any media. Incident photons are annihilated in these processes, giving rise to scattered photons at redder Stokes or bluer anti-Stokes frequencies. These scattering events lead to the creation or annihilation of phonons, respectively. Although previous research in optomechanics has used bolometric forces ${ }^{9-11}$, centrifugal radiation pressure ${ }^{12-18}$ and optical gradient force $e^{19-21}$ to excite mechanical motion of device boundaries, it is only recently that electrostrictive Brillouin scattering ${ }^{5-7}$ was demonstrated in microcavities. As electrostriction is one aspect of radiation pressure, extensive work in radiation pressure cooling ${ }^{13-18}$ raises the question of whether it is possible to cool phonon modes by means of a Brillouin process, similar to the scattering process available in bulk media ${ }^{1}$. For achieving Brillouin anti-Stokes cooling, the heating Stokes line needs to be eliminated. However, as low acoustic frequencies separate the pump line from the Stokes and anti-Stokes lines, filtering out the Stokes line against the anti-Stokes line requires a rapid transmission change over an extremely small frequency difference, which is not easily available in bulk materials. Here we transform the energy flow direction in spontaneous Brillouin scattering using two discrete optical resonances separated by the mechanical-resonance frequency. This allows the Brillouin cooling process to gain efficiency and selectivity by pumping at the lower resonance and emitting anti-Stokes photons at the upper resonance. This is different from systems with a single optical resonance, where cooling was achieved by detuning the pump below the resonance by a distance equal to the frequency of the mechanical mode ${ }^{22}$. Furthermore, in contrast to boundary-deforming optomechanical systems ${ }^{10,12-18}$ where the radial vibration can leak through the support, the whispering-gallery mechanical mode ${ }^{7}$ here propagates azimuthally along the device equator. This orthogonal relationship reduces anchoring-related dissipation, and thus the mechanical quality factor here can approach the material limit ${ }^{1}$.

Our room-temperature and atmospheric-pressure experiment (Fig. 1c) is based on a silica microsphere resonator ${ }^{23}$ of $Q_{\text {optical }}>10^{8}$. We evanescently couple light into the resonator by means of a tapered optical fibre ${ }^{24}$. Light is coupled out from the other side of this taper, allowing interrogation of the acoustical mode by means of the light that is scattered from it. This spherical resonator supports the three participants in the Brillouin cooling process - two optical resonances (pump and anti-Stokes) and one acoustical resonance. The acoustical and the optical modes are of a whispering-gallery type and circulate in unison with considerable overlap. These three modes are coupled by means of photoelastic scattering induced by density change (Supplementary Equations (S9)-(S11)), and the electrostrictive effect (Fig. 1a and Supplementary Equation (S24)), that are two facets of one single mutual coupling defined by the electrostrictive constant, $\gamma_{e}$, of the material. Experimental interrogation of the acoustical mode is made possible through observations of the pump light that it scatters (and Doppler shifts) into the anti-Stokes mode. The beat note between the pump light and the anti-Stokes light is measured on a photodetector at the output of the taper and is a measurement of the acoustical mode. The mechanical deformation of the sphere is illustrated with 12 acoustical wavelengths along the circumference in Fig. 1c. This illustration corresponds to an experimentally observed (Figs 2-4) acoustical mode of frequency $\Omega_{\mathrm{a}}=95 \mathrm{MHz}$ on a $77.5-\mu \mathrm{m}$-radius sphere, which we numerically calculate in the Supplementary Information. In this work, we will experimentally cool this mechanical mode.

Although energy considerations give a general explanation for cooling, an explicit solution is needed. We therefore also analytically derive the cooling process while starting from the acoustical- and optical-wave equations in the Supplementary Information. The source terms in these equations are taken to be electrostrictive pressure for the acoustical wave, and photoelastic index change for the optical wave. As expected from energy considerations, the solution shows that light applies compressive pressure on the region of the acoustical wave that expands, to take away energy from sound. We also confirm that repeating the same calculation for the Stokes process gives pressure on the acoustical regions while they shrink so that energy is imparted to the acoustical wave as expected. The classical analysis also shows that higher acoustical and optical quality factors, and lower acoustical frequencies, improve the cooling ratio (Supplementary Equation (S44)). A quantum analysis ${ }^{25}$ provides more details on the feasibility of this system towards ground-state cooling ${ }^{17,18,26}$, and is used to estimate the optomechanical coupling strength $g_{0}$ in Supplementary Section S5. The principal enablers

${ }^{1}$ Electrical Engineering and Computer Science, University of Michigan, Ann Arbor, Michigan 48109, USA, ${ }^{2}$ Institut für Theoretische Physik, Universität Erlangen-Nürnberg, Staudtstrasse 7, D-91058 Erlangen, Germany, ${ }^{3}$ Max Planck Institute for the Science of Light, Günther-Scharowsky-Strasse 1/Bau 24, D-91058 Erlangen, Germany. *e-mail: bahlg@umich.edu. 
a

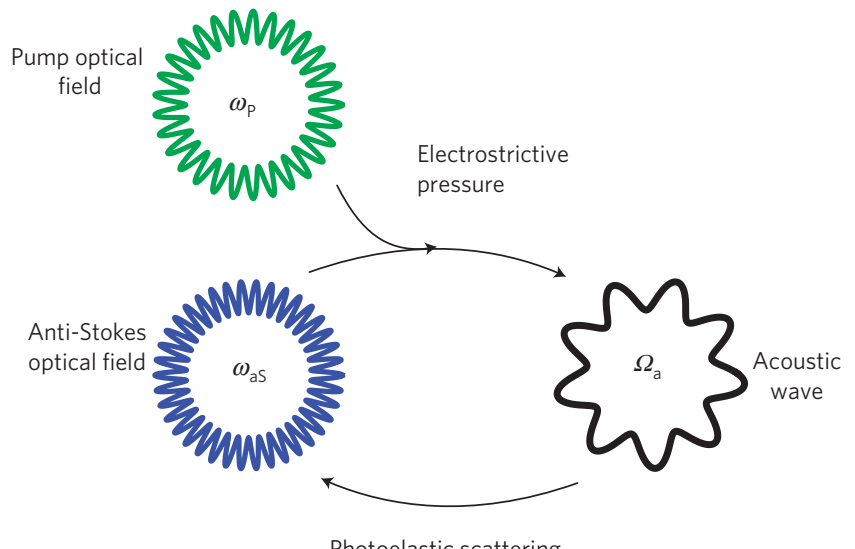

b
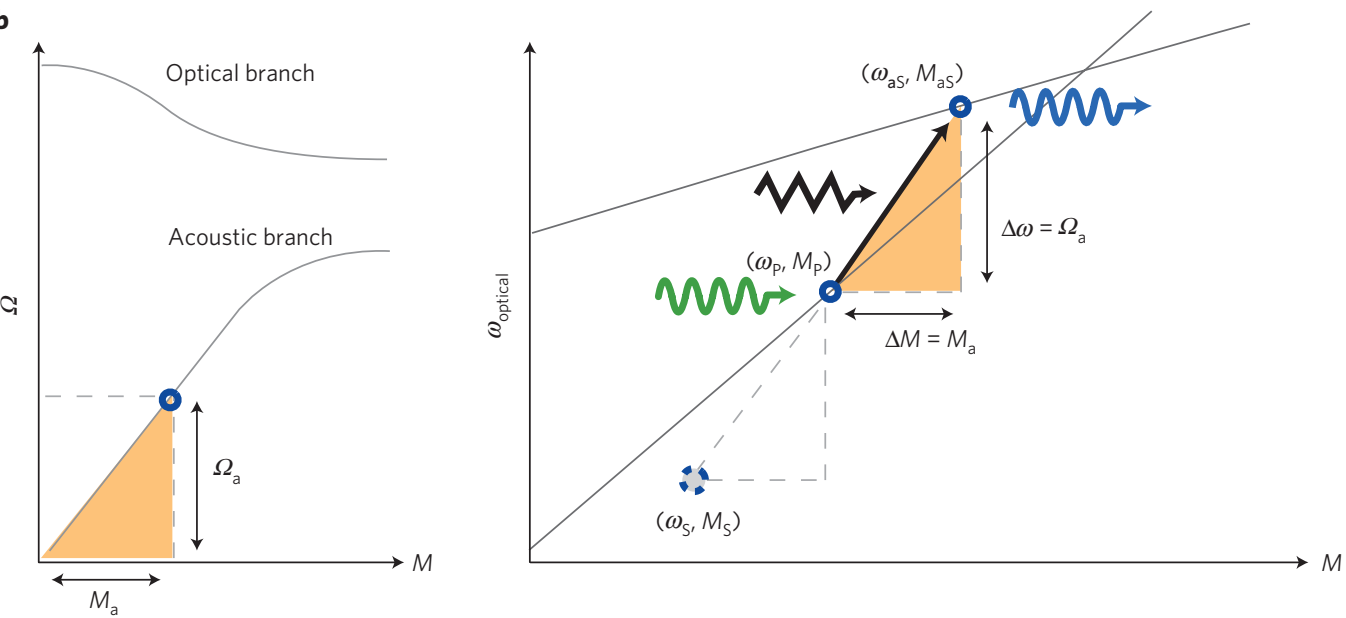

C

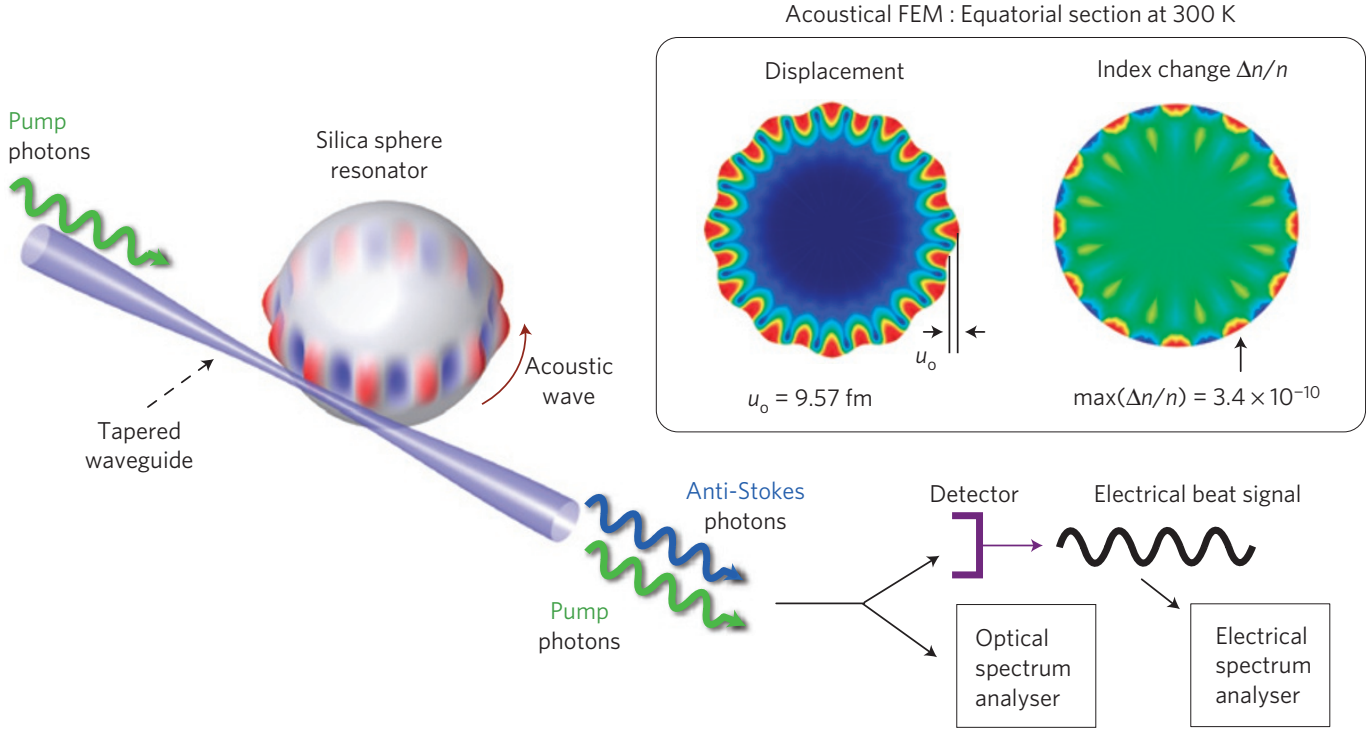

Figure 1 | Conceptual description of Brillouin cooling. a, Cooling of an acoustic wave through the interplay between anti-Stokes photoelastic scattering and electrostrictive pressure. $\mathbf{b}$, Selectively enabling Brillouin anti-Stokes cooling (a) while rejecting Stokes heating is achieved by relying on a resonator with asymmetric optical resonance structure (right) that matches the energy and momentum of the acoustical mode being cooled (left). c, Pump photons photoelastically scatter from the Brownian acoustical mode, and thermal energy is removed because only the anti-Stokes process is resonantly enhanced. Inset: finite-element method (FEM) calculation of an acoustical mode with 12 wavelengths around the circumference, which corresponds to the frequency $\Omega_{\mathrm{a}}=95 \mathrm{MHz}$ on a $77.5-\mu \mathrm{m}$-radius sphere. The amplitude and index perturbation are calculated for $300 \mathrm{~K}$.

for Brillouin cooling are: the selective resonant enhancement of the anti-Stokes cooling process over the Stokes process; and phase matching for the two optical modes and the acoustic mode interacting through the Brillouin process (Methods and Fig. 1b).
Experimental measurement of Brillouin cooling is shown in Fig. 2, left, as indicated by the broadening of the beating signal between the pump and anti-Stokes lines, as a function of increasing pump power. This beat observed from the tapered fibre coupler 

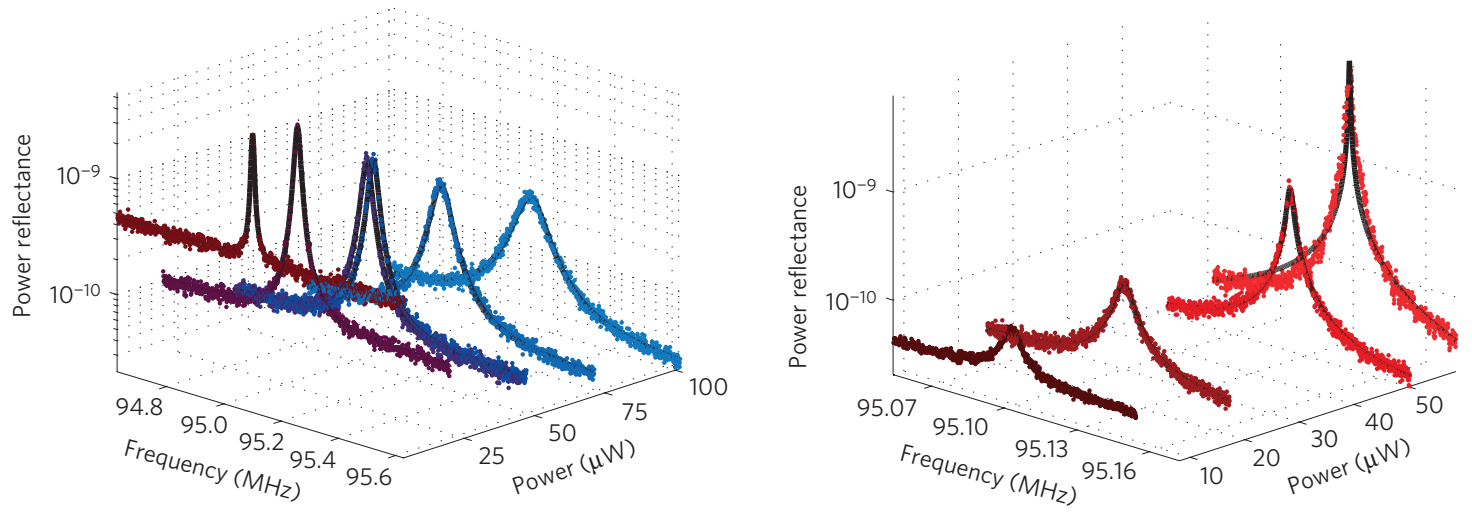

Figure 2 | Experimental observation of cooling and heating of a $95 \mathrm{MHz}$ acoustical mode in the microsphere system. The spectra of power reflectance from the density-wave fluctuation are provided as a function of input optical power. The fact that the area under the Lorentzian curves becomes smaller at high input power while the linewidth broadens indicates that cooling is occurring. To cool the acoustical mode (left), we pump the low-frequency optical resonance $\left(O_{p}\right)$ to only resonantly enhance the anti-Stokes process while suppressing the Stokes process. For the heating experiment (right), we pump the higher-frequency optical resonance $\left(\mathrm{O}_{\mathrm{as}}\right)$ instead, to resonantly enhance the Stokes process. The noise floor drops at high optical power because the measurement is performed with more pump light inside the cavity for which a normalization is carried out.
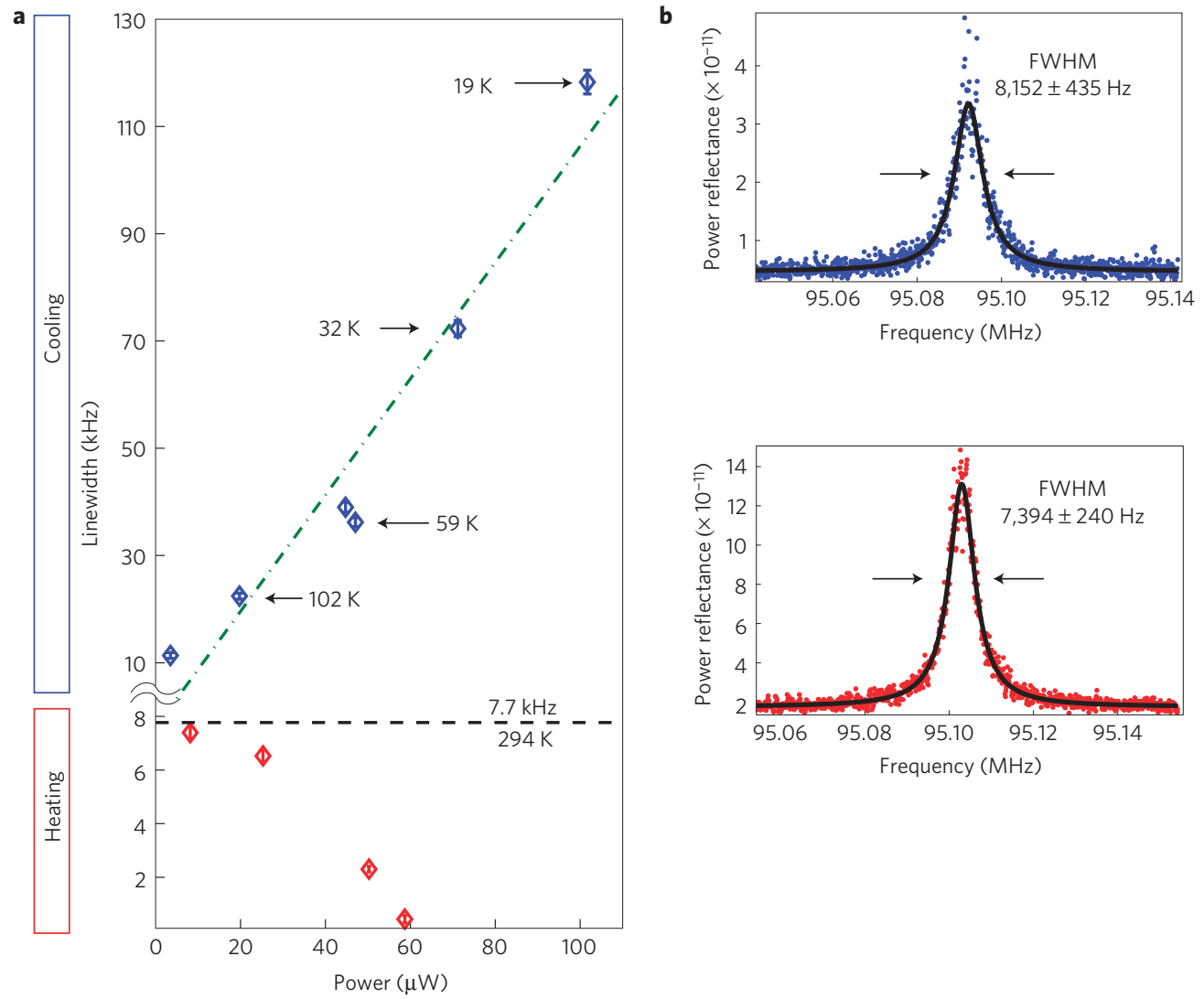

Figure 3 | Experimental measurement of acoustical mode temperature. a, We capture linewidth data from a cooling and heating experiment, and calculate the effective acoustical mode temperatures. As expected, both cooling and heating linewidths converge towards the Brownian room-temperature linewidth at low optical pump power. The green dash-dot line is a fit to the theory in Supplementary Equation (S44). Error bars indicate the 95\% confidence bounds for the measured linewidth. $\mathbf{b}$. We calibrate the acoustical linewidth as a function of temperature by finding the point of convergence of the cooling and heating data. The narrowest observed cooling linewidth (top) and the widest observed heating linewidth (bottom) are averaged to estimate the $7.7 \mathrm{kHz}$ linewidth for the acoustical mode at ambient temperature of $294 \mathrm{~K}$. FWHM, full-width at half-maximum.

serves as a measurement of the acoustical mode (see Supplementary Information). The cooling experiment is carried out by positioning the pump laser at the lower-frequency resonance ( $\omega_{\mathrm{P}}$ in Fig. 1b), and by observing the light scattered in the anti-Stokes direction into the $\omega_{\mathrm{as}}$ resonance. As in refs 10,13, we prefer linewidth as a measurement of cooling ${ }^{10,13-18,26-28}$ because the alternative of measuring the integrated power of the beat note might be affected by attenuation of the optical signals. As the power reflectance spectrum is proportional to the squared displacement spectrum of the acoustical mode (see Supplementary Information), the acoustical linewidth has been extracted from this data and is presented in Fig. 3a. Here, the linewidth of the $95 \mathrm{MHz}$ acoustical 


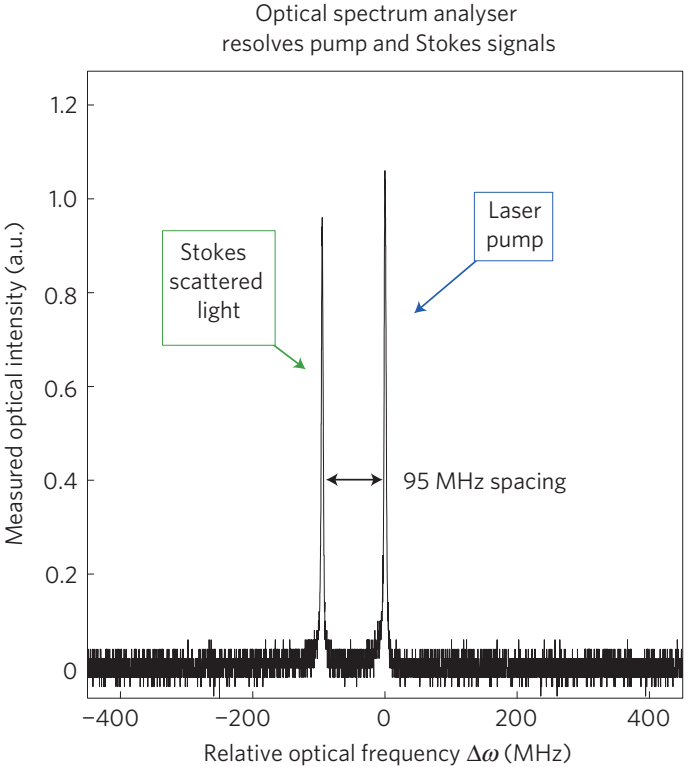

b

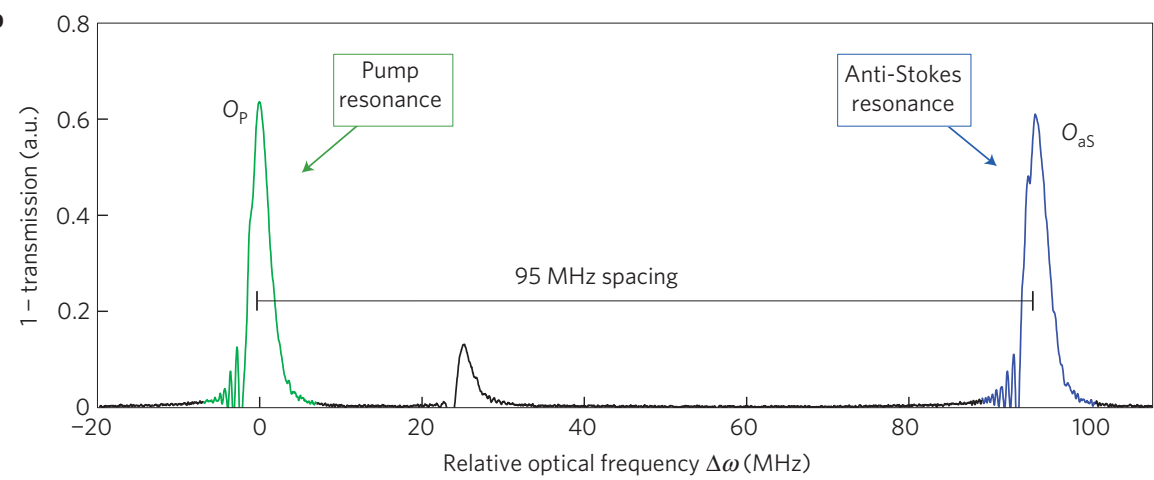

Figure 4 | Experimental verification of phase matched optical and acoustical modes. a, The participating resonances are analysed by three independent experimental measurements, confirming that the optical signals beating on the photodetector (right) indeed originate from one pump and one Stokes

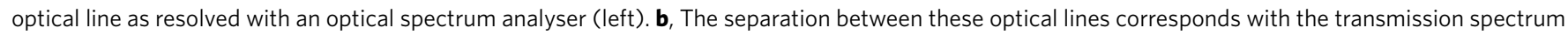
of our device, measured by sweeping the pump wavelength. Absorption peaks that overlap the lines resolved in a (left) are seen. Resonances are named according to their role in the cooling experiments.

mode increases from $7.7 \mathrm{kHz}$ to $118 \mathrm{kHz}$ with increasing pump power, in an open laboratory environment at $294 \mathrm{~K}$. We measure the effective mode temperature through its inverse proportionality to the linewidth of the acoustical mode (see Supplementary Information). The result indicates an achieved cooling ratio of 15 , implying an effective mode temperature of $19 \mathrm{~K}$ at the maximum observed cooling point. The cavity operates in the thermally stable regime $^{29}$. The cooling factor we achieved was limited by the optical power available from the pump laser. To complete the analysis, we now invert this process by moving the pump to the higher-frequency resonance. The low-frequency optical mode now functions as a Stokes resonance for the higher-frequency pump. As expected, line narrowing is now observed as indicated in Fig. 3a, accompanied by growth of the Stokes signal (Fig. 2, right). The Stokes process linewidth and the anti-Stokes cooling linewidth converge at low input powers, indicating the mechanical quality factor of the mode. This point of convergence provides the $7.7 \mathrm{kHz}$ acoustical linewidth calibration at $294 \mathrm{~K}$ in Fig. 3, so that mode temperature can be calculated during the cooling experiment. On the basis of the frequency-Q product reported for silica ${ }^{1}$, one can calculate a material-limited mechanical Q to be 7 times higher than what we measured here. We believe that transitioning to vacuum, where air dissipation plays no role, will allow getting closer to the material-limited Q.
We experimentally confirm that two optical resonances exist that are separated by the mechanical frequency, as required for phase-matched cooling. We already know that the pump to anti-Stokes beat, measured by a photodetector, provides us with a frequency of $95 \mathrm{MHz}$ for the mechanical mode (Fig. 4a, right). We then carry out the following two independent measurements that obtain no information from this electrical measurement. Measuring resonator transmission while scanning the pump frequency (Fig. 4b) indeed confirms the existence of two optical resonances spaced by $95 \mathrm{MHz}$ to within measurement error. We also verify that the relevant resonance structure is indeed asymmetric relative to the pump by confirming that no resonance appears on the Stokes side of pump resonance $O_{\mathrm{P}}$ at a spacing of $95 \mathrm{MHz}$. The optical line frequency spacing is also measured by repeating the Stokes experiment ${ }^{7}$ but now with an optical spectrum analyser at the output. As expected, the spectrum analyser resolves two optical lines that are separated by $95 \mathrm{MHz}$ (Fig. 4a, left) in agreement with the measured transmission profile. These three independent measurementsbeat spectrum, optical transmission and the optical output spectrum-confirm the existence of the resonant triplet that was used in the cooling process.

Here we use forward Brillouin scattering ${ }^{3,7}$ in a system involving two optical modes and one acoustical mode to demonstrate 
cooling. In comparison, previous optical cooling studies have used fluorescence ${ }^{30}$, radiation pressure forces ${ }^{13-18}$ and photothermal pressure $^{10}$. Although the end result here combines a net annihilation of phonons ${ }^{10,13-18,26,30}$ in a multi-resonance system ${ }^{5-8}$, no detuning of the pump with respect to the resonance is required for achieving cooling. Furthermore, the fact that the mechanical mode is of a whispering-gallery type here, indicates low dissipation through the support ${ }^{7}$. Although we selected the $95 \mathrm{MHz}$ mode in this study because of the greater cooling ratio achievable for such relatively low frequencies (Supplementary Equation (S44)), mechanical modes spanning from tens of megahertz ${ }^{7}$ to tens of gigahertz ${ }^{5,6}$ can be accessed with this method. In a broad context, Brillouin scattering belongs to a family of scattering processes that includes Raman and Rayleigh scattering. Reversing the energy flow direction here, with respect to Brillouin systems such as those used in refs 3,5-7, raises the question of whether similar inversion is possible with Raman scattering ${ }^{31}$ nonoptomechanical cooling of solids $s^{30,32,33}$ in spite of the higher frequencies involved.

\section{Methods}

Phase matching and selective resonant enhancement of the anti-Stokes process. Selective resonant enhancement of the anti-Stokes process (Fig. 1a) is made possible owing to the non-periodic frequency separation ${ }^{34,35}$ of the high-order optical resonances of the sphere. Here we exploit this non-periodic resonance structure for solely selecting the anti-Stokes line. The phase-matching requirement implies that the anti-Stokes photon should carry out the energy $(\omega)$ and momentum $(M)$ of the pump photon and the thermal phonon. In a spherical resonator, the momentum parameter $M$ relates to azimuthal propagation $\mathrm{e}^{i(M \phi-\omega t)}$ around the sphere equator. As expected, these conservation consideration ${ }^{36}$ are in agreement with our analytically derived synchronous solution for the coupled-wave equations ${ }^{1}$ that describe our system (see Supplementary Information). Both phase matching and selective resonant enhancement are illustrated in the energy-momentum $(\omega-M)$ diagram of Fig. 1b. As the pump optical resonance $\left(\omega_{\mathrm{P}}, M_{\mathrm{P}}\right)$ and the anti-Stokes resonance $\left(\omega_{\mathrm{aS}}, M_{\mathrm{aS}}\right)$ are separated by the acoustical resonance parameters $\left(\Omega_{\mathrm{a}}, M_{\mathrm{a}}\right)$, as indicated by the filled triangles, both phase matching and resonant enhancement are satisfied for anti-Stokes scattering. On the other hand, the dashed triangle of Fig. $1 \mathrm{~b}$ illustrates the off-resonantly eliminated Stokes process, where subtraction of the same acoustical parameters $\left(\Omega_{\mathrm{a}}, M_{\mathrm{a}}\right)$ from the pump resonance $\left(\omega_{\mathrm{p}}, M_{\mathrm{P}}\right)$ brings us to a region $\left(\omega_{S}, M_{S}\right)$ where no optical resonances exist.

Received 9 September 2011; accepted 9 December 2011; published online 22 January 2012

\section{References}

1. Boyd, R. W. Nonlinear Optics 3rd edn, Ch. 9 (Elsevier, 2008).

2. Chiao, R. Y., Townes, C. H. \& Stoicheff, B. P. Stimulated Brillouin scattering and coherent generation of intense hypersonic waves. Phys. Rev. Lett. 12, 592-595 (1964).

3. Shelby, R., Levenson, M. \& Bayer, P. Resolved forward Brillouin scattering in optical fibers. Phys. Rev. Lett. 54, 939-942 (1985).

4. Dainese, P. et al. Stimulated Brillouin scattering from multi-GHz-guided acoustic phonons in nanostructured photonic crystal fibres. Nature Phys. 2, 388-392 (2006).

5. Grudinin, I. S., Matsko, A. B. \& Maleki, L. Brillouin lasing with a $\mathrm{CaF}_{2}$ whispering gallery mode resonator. Phys. Rev. Lett. 102, 043902 (2009).

6. Tomes, M. \& Carmon, T. Photonic micro-electromechanical systems vibrating at X-band (11-GHz) rates. Phys. Rev. Lett. 102, 113601 (2009).

7. Bahl, G., Zehnpfennig, J., Tomes, M. \& Carmon, T. Stimulated optomechanical excitation of surface acoustic waves in a microdevice. Nature Commun. 2 , 403 (2011).

8. Grudinin, I. S., Lee, H., Painter, O. \& Vahala, K. J. Phonon laser action in a tunable two-level system. Phys. Rev. Lett. 104, 083901 (2010).

9. Mertz, J., Marti, O. \& Mlynek, J. Regulation of a microcantilever response by force feedback. Appl. Phys. Lett. 62, 2344-2346 (1993).

10. Metzger, C. \& Karrai, K. Cavity cooling of a microlever. Nature 432, 1002-1005 (2004).

11. Metzger, C. et al. Self-induced oscillations in an optomechanical system driven by bolometric backaction. Phys. Rev. Lett. 101, 133903 (2008).

12. Carmon, T., Rokhsari, H., Yang, L., Kippenberg, T. \& Vahala, K. Temporal behavior of radiation-pressure-induced vibrations of an optical microcavity phonon mode. Phys. Rev. Lett. 94, 223902 (2005).
13. Arcizet, O., Cohadon, P-F., Briant, T., Pinard, M. \& Heidmann, A.

Radiation-pressure cooling and optomechanical instability of a micromirror. Nature 444, 71-74 (2006).

14. Gigan, S. et al. Self-cooling of a micromirror by radiation pressure. Nature 444, 67-70 (2006).

15. Kleckner, D. \& Bouwmeester, D. Sub-kelvin optical cooling of a micromechanical resonator. Nature 444, 75-78 (2006).

16. Thompson, J. et al. Strong dispersive coupling of a high-finesse cavity to a micromechanical membrane. Nature 452, 72-75 (2008).

17. Chan, J. et al. Laser cooling of a nanomechanical oscillator into its quantum ground state. Nature 478, 89-92 (2011).

18. Riviere, R. et al. Optomechanical sideband cooling of a micromechanical oscillator close to the quantum ground state. Phys. Rev. A 83, 063835 (2011).

19. Povinelli, M. L. et al. Evanescent-wave bonding between optical waveguides. Opt. Lett. 30, 3042-3044 (2005).

20. Li, M. et al. Harnessing optical forces in integrated photonic circuits. Nature 456, 480-484 (2008).

21. Eichenfield, M., Camacho, R., Chan, J., Vahala, K. J. \& Painter, O. A picogramand nanometre-scale photonic-crystal optomechanical cavity. Nature 459, 550-555 (2009).

22. Schliesser, A., Riviere, R., Anetsberger, G., Arcizet, O. \& Kippenberg, T. Resolved-sideband cooling of a micromechanical oscillator. Nature Phys. 4, 415-419 (2008).

23. Gorodetsky, M \& Ilchenko, V. High-Q optical whispering-gallery microresonators: Precession approach for spherical mode analysis and emission patterns with prism couplers. Opt. Commun. 113, 133-143 (1994).

24. Knight, J., Cheung, G., Jacques, F. \& Birks, T. Phase-matched excitation of whispering-gallery-mode resonances by a fiber taper. Opt. Lett. 22, 1129-1131 (1997).

25. Tomes, M., Marquardt, F., Bahl, G. \& Carmon, T. Quantum mechanical theory of optomechanical Brillouin cooling. Phys. Rev. A 84, 063806 (2011.)

26. Teufel, J. D. et al. Sideband cooling of micromechanical motion to the quantum ground state. Nature 475, 359-363 (2011).

27. Naik, A. et al. Cooling a nanomechanical resonator with quantum back-action. Nature 443, 193-196 (2006).

28. Kippenberg, T. J. \& Vahala, K. J. Cavity optomechanics: Back-action at the mesoscale. Science 321, 1172-1176 (2008).

29. Carmon, T., Yang, L. \& Vahala, K. Dynamical thermal behavior and thermal self-stability of microcavities. Opt. Express 12, 4742-4750 (2004).

30. Epstein, R. \& Sheik-Bahae, M. (eds) Optical Refrigeration 1st edn (Wiley-VCH, 2009).

31. Kang, M. S., Nazarkin, A., Brenn, A. \& Russell, P. S. J. Tightly trapped acoustic phonons in photonic crystal fibres as highly nonlinear artificial Raman oscillators. Nature Phys. 5, 276-280 (2009).

32. Vermeulen, N., Debaes, C., Muys, P. \& Thienpont, H. Mitigating heat dissipation in Raman lasers using coherent anti-stokes Raman scattering. Phys. Rev. Lett. 99, 093903 (2007).

33. Jalali, B. Laser design: A cooler Raman laser. Nature Photon. 1, 691-692 (2007).

34. Savchenkov, A. A., Matsko, A. B., Ilchenko, V. S., Strekalov, D. \& Maleki, L. Direct observation of stopped light in a whispering-gallery-mode microresonator. Phys. Rev. A 76, 023816 (2007).

35. Carmon, T. et al. Static envelope patterns in composite resonances generated by level crossing in optical toroidal microcavities. Phys. Rev. Lett. 100, 103905 (2008).

36. Ding, L. et al. High frequency GaAs nano-optomechanical disk resonator. Phys. Rev. Lett. 105, 263903 (2010).

\section{Acknowledgements}

This work was supported by the Defense Advanced Research Projects Agency (DARPA) Optical Radiation Cooling and Heating in Integrated Devices (ORCHID) programme through a grant from the Air Force Office of Scientific Research (AFOSR). M.T. is supported by a National Science Foundation fellowship. F.M. acknowledges the Emmy-Noether programme.

\section{Author contributions}

G.B., M.T. and T.C. designed and conceived the experiments. G.B. carried out the experiments and numerical calculations. All authors jointly carried out the analytical calculations, analysed the data and co-wrote the paper. T.C. supervised all aspects of this project.

\section{Additional information}

The authors declare no competing financial interests. Supplementary information accompanies this paper on www.nature.com/naturephysics. Reprints and permissions information is available online at www.nature.com/reprints. Correspondence and requests for materials should be addressed to G.B. 